\title{
Now Perceiving: The Complete Genome Package
}

\section{Tapasya Srivastava*}

University of Delhi South Campus, India

*Corresponding author: University of Delhi South Campus, New Delhi, India, Tel- 91-11-24112761; E-mail: tapasya@south.du.ac.in

Received date: December 12, 2014, Accepted date: December 18, 2014, Published date: December 24, 2014

Copyright: (c) 2014 Srivastatva. This is an open-access article distributed under the terms of the Creative Commons Attribution License, which permits unrestricted use, distribution, and reproduction in any medium, provided the original author and source are credited.

\section{Editorial}

The genetic basis of cancer has expanded from studying individual gene and molecules to using a systems biology approach in deciphering the onset and development of the disease. The biology of the disease has revealed itself to be more complex than the initial 'Hallmarks of cancer' as defined by Hanahan and Weinberg in 2000, wherein the activation of proliferation, metastasis and angiogenesis along with signs of resistance to death and evasion of tumor suppressors, co-exist. Since then a large body of work has elucidated the role of immune cells, inflammation and metabolism within the tumor to be an equal contributing in the genesis and progression of this heterogeneous disease. Cells and molecules in the stroma together comprise the tumor microenvironment and these are known to activate signaling cascades, which help in tumor growth and progression. Cancer development itself has changed its fate as and when new discoveries of tumor suppressor genes, oncogenes and cell types have been validated. The concept of a linear progression of cancer has been significantly altered by the discovery of regulatory molecules, which modulate the driver and passenger mutation in a cell.

Identification of these mutations in cancer gene has acquired new proportions with the technological advances. Large datasets and careful compartmentalization of tumor and non-tumor cells have generated consensus and dependable information, which when analyzed, identifies genes whose function and mechanism can be further deciphered. From a morphological basis of classification of tumors, analysis of these datasets have now put forth a classification based on the genetic alteration observed in a tumor. The initial Knudson's 'two-hit hypothesis' and Loeb's 'mutator phenotype' had formed the premises of understanding genetic changes in a cancer cell. The recent $\mathrm{M}$ (mutation) and C (copy number) classification given by Chris Sander's group at Sloan-Kettering have observed that the two types of alterations usually do not overlap in the 12 cancers they have tried to classify based on 500 functional events, including epigenetic changes. The process of separating the basal mutation rate, which may occur due to the environment or other random events, involves developing algorithms based on baseline data from normal tissue. A cancer genome involves some driver mutations, which can initiate tumorigenesis, and a bunch of passenger mutations that are neutral in behavior and are carried along with an evolved cancer cell as and when it progresses. Most efforts while analyzing these datasets are towards removing the passenger mutations to distinctly predict the driver mutations as the primary event of cancer formation. However, one must bear in mind that the passenger mutations of the surviving cell may play an important role in the subsequent metastasizing cell. Or even in a different sub-population of clonal cells emerging at the onset of tumorigenesis. Targeted therapy for cancers have mostly addressed the driver mutations of the tumorigenic process, however in a growing tumor and metastasizing cells, these may not always be the relevant target. Intra-tumor heterogeneity in a tumor is an indicator of both the origin as well as the clonality of the cell population in the tumor cell and has emerged as a major deterrent of targeted therapy. The epigenetic layer of regulatory molecules delves into the complexity of the genome and methylation patterns have emerged as potential biomarkers. Essentially, biomarker discovery for early detection of cancers and genetic counseling for familial cancers, still remains a hope for prevention, but not corrected.

Identifying a mutation spectrum as a genetic basis for a particular cancer has been the biggest outcome of the concerted effort of cancer geneticist around the world who undertook the effort of providing quality data from sequence analysis. For this, scientists have used biological material ranging from blood, tissue samples to circulating tumor cells. These have provided invaluable information on the existing intra tumor heterogeneity and the difficulties it presents in molecular characterization of the tumor. These high-throughput data allows researchers to have the confidence of attempting to categorize tumors for stratified medicine. As opposed to the empirical medicines, which did not consider one individual as any different from the other, the stratified medicines include testing of at least one biomarker to be able to assess the potency and efficacy of a drug. Hence, analyzing the genetic heterogeneity of a tumor compartment to understand the growth and clonality of a tumor would offer a repertoire of tumor biomarkers that may be targeted for therapy. The role of tumor microenvironment in contributing to this heterogeneity is undeniably the prime focus of current research work. A tumor microenvironment strongly determines the growth and aggressiveness of a tumor as the stroma and all the associated cell types offer a dynamic environment to the tumor epithelial cells to adapt and grow. The last decade has seen a surge in the research work on the immune cells, inflammatory response and hypoxic tumor microenvironment. An interesting interpretation of tumor progression under the influence of tumor microenvironment implicates the formation of a small number of metastatic cell types different from those leading to the primary tumor formation. This would explain the reason why some tumors are highly aggressive in their metastatic behavior.

This also sets precedence for development of appropriate functional models of cancer to study their biology as well as for development of therapeutic drugs. A mechanistic understanding of how genetic mutations and alterations can affect the prognosis of a disease and its treatment regimen, requires identification of associated genes as well as their functional analysis. Stable cell-lines, tissue derived cell-lines and various mouse models have together been the mainstay of such derivations. Studies on tumor microenvironment reveal how its influence can drastically change the dynamics of cell signaling. The drawback of using cell-lines for pre-clinical drug testing, without appropriate conditions which mimic the tumor microenvironment have compromised on their potential use in vivo. However, they offer a distinct advantage when patient derived cell-lines from biopsy or primary tumor tissue allows us to check an individual patient for drug efficacy before the treatment begins. Influence of a tumor 
Page 2 of 2

microenvironment can be studied by using cell-lines in hypoxia and starvation as well as in mouse models targeting molecules of key pathways, such as hypoxia and angiogenesis. Orthotropic and xenografts models are also frequently used as a relatively easier and quicker option. Using genetically engineered mouse models using forward and reverse genetics in conditional mice, reveals the most comprehensive information on tumor biology.
There is a compounding need to enhance and substantiate the flurry of information obtained by the technically advanced highthroughput methods in cancer genetics with appropriate functional models. This would help in interpreting the regulatory and interactive gene-to-gene as well as cell-to-cell networks in a tumor microenvironment, and facilitate development of better therapeutic modalities.

This article was originally published in a special issue, entitled: "Cancer Biology and Therapeutice", Edited by Tapasya Srivastatva 